\title{
Testing extra dimensions hypothesis in high energy physics
}

\author{
I. Volobuev* \\ SINP MSU \\ E-mail: volobuev@theory.sinp.msu.ru \\ E. Boos \\ SINP MSU \\ E-mail: boosetheory.sinp.msu.ru

\section{Bunichev} \\ SINP MSU \\ E-mail: bunichev@theory.sinp.msu.ru
}

M. Perfilov

SINP MSU

E-mail: perfilovefnal.gov

\section{Smolyakov}

SINP MSU

E-mail: smolyakov@theory.sinp.msu.ru

The history of the hypothesis about extra dimensions is reviewed and the physics and phenomenology of models with large extra dimensions are discussed with an emphasis on the RandallSundrum model with two branes. We argue that the original Randall-Sundrum model is phenomenologically unacceptable, because the massless radion couples too strongly to matter on the negative tension brane, and should be stabilized. For the stabilized Randall-Sundrum model, the physical degrees of freedom are explicitly isolated. The problem of constructing brane world models with more than one extra dimensions is touched upon. The phenomenology of the stabilized Randall-Sundrum model is discussed in detail and specific changes of the distribution tails for the processes of SM particles scattering at the Tevatron and the LHC due to the contribution of the KK tower are found. We also consider the case of the universal extra dimensions, where the SM gauge bosons can propagate in the bulk, and present the cross-sections of the single top and Drell-Yan production processes taking into account the contributions of the Standard Model gauge bosons, of their first KK modes and of the corresponding KK towers and discuss the impact of the interference between them. For pp-collisions at the LHC with the center of mass energy $14 \mathrm{TeV}$ we also found changes of the distribution tails due to the interference effects. Such a modification of distribution tails is characteristic for the processes mediated by particles coming from extra dimensions and should always be taken into account when looking for them.

The XXI International Workshop High Energy Physics and Quantum Field Theory,

June 23 - June 30, 2013

Saint Petersburg Area, Russia

\footnotetext{
* Speaker.
} 


\section{Introduction}

The hypothesis about extra dimensions of space-time that was put forward almost a century ago has been especially widely discussed in theoretical physics during the last decades. There is a good reason to believe that this hypothesis stems from the ideas formulated by Bernhard Riemann in his famous lecture "Über die Hypothesen, welche der Geometrie zu Grunde liegen" (1854) (On the Hypotheses which lie at the Bases of Geometry) [1]:

The questions about the infinitely great are for the interpretation of nature useless questions. But this is not the case with the questions about the infinitely small. It is upon the exactness with which we follow phenomena into the infinitely small that our knowledge of their causal relations essentially depends....

Now it seems that the empirical notions on which the metrical determinations of space are founded, the notion of a solid body and of a ray of light, cease to be valid for the infinitely small. We are therefore quite at liberty to suppose that the metric relations of space in the infinitely small do not conform to the hypotheses of geometry; and we ought in fact to suppose it, if we can thereby obtain a simpler explanation of phenomena....

This leads us into the domain of another science, of physics, into which the object of this work does not allow us to go today.

In this lecture, the notion of n-dimensional space was formulated, which suggested that one of the possible ways to modify the space-time geometry at small distances was to assume the existence of extra dimensions.

A space-time with extra dimensions first appeared in theoretical physics back in 1914, as Gunnar Nordström made an attempt to unify a relativistic scalar gravity theory that he was developing at that time with electrodynamics in his work "Über die Möglichkeit, das elektromagnetische Feld und das Gravitationsfeld zu vereinigen" (On the possibility of unifying the electromagnetic and the gravitational fields) [细. In Nordström's approach, our four-dimensional world was embedded in a five-dimensional one as a submanifold, which was very similar to the present-day brane-world models. Though Nordström's scalar theory of gravity was a very important step in constructing Einstein's general theory of relativity, it was forgotten soon after the latter proved to be a success. The idea of extra dimensions was also forgotten for five years to be rediscovered by Theodor Kaluza and implemented in the framework of Einstein's general theory of relativity.

\section{Kaluza-Klein theory}

In his famous paper [3], T. Kaluza considered a pure gravity with the standard Einstein equations in a five-dimensional space-time of the form $E=M^{4} \times R^{1}$, where $M^{4}$ is the four-dimensional space-time. If we denote the coordinates in $E$ by $X^{N}=\left(x^{v}, y\right), \quad N=0,1, \cdots 4, v=0,1, \cdots 3$, the corresponding gravitational action in this space can be written as

$$
S=\frac{1}{16 \pi \hat{G}} \int_{E} \hat{R} \sqrt{-g} d^{5} X
$$

where $\hat{G}$ is the five-dimensional gravitational constant and $\hat{R}$ is the scalar curvature of the metric $g_{M N}, \operatorname{sign}(g)=(-,+\cdots+)$. This is the most natural extension of the gravitational action to a 
theory with extra dimensions, but not the only one possible. Below we will touch upon other possible extensions of the gravitational action, which lead to different properties of multidimensional gravity.

T. Kaluza decomposed the five-dimensional metric $g_{M N}$ into the four-dimensional part and the extra components as follows:

$$
g_{M N}=\left(\begin{array}{cc}
g_{\mu \nu} & 2 \alpha A_{\mu} \\
2 \alpha A_{v} & 2 \phi
\end{array}\right), \quad 2 \alpha A_{\mu}=g_{\mu 4}, 2 \phi=g_{44} .
$$

In the case where $g_{M N}$ does not depend on $y$, which Kaluza called the "cylindricity condition", the scalar curvature $\hat{R}$ can be expressed as

$$
\begin{aligned}
\hat{R} & =R_{(4)}-\frac{\alpha^{2}}{2} F_{\mu \nu} F^{\mu v} \phi^{-1}-\frac{1}{2} \phi^{-2} \partial_{\mu} \phi \partial^{\mu} \phi \\
F_{\mu v} & =\partial_{\mu} A_{v}-\partial_{v} A_{\mu},
\end{aligned}
$$

$R_{(4)}$ being the scalar curvature of the four-dimensional space-time $M^{4}$ with metric $g_{\mu \nu}$.

In his paper, T. Kaluza interpreted the field $A_{\mu}$ as the electromagnetic field. But the subsequent studies showed that this interpretation failed, and even the original ansatz for the five-metric was inconsistent.

The idea of T. Kaluza was later developed by O. Klein [4], who suggested to explain the unobservability of the fifth dimension by its compactness and extremely small size of the order of the Planck length. In this case the five-dimensional space-time looks like $E=M^{4} \times S^{1}$, where $S^{1}$ is the circle of circumference $L$.

He proposed to consider the vacuum configuration of the five-dimensional metric and to include scalar, spinor and vector fields in the multidimensional theory from the very beginning.

He also pointed out that free fields in five-dimensional space-time satisfy the five-dimensional Klein-Gordon equation

$$
\left(\partial_{M} \partial^{M}-m^{2}\right) \phi=0,
$$

and that any five-dimensional field in the space-time $E=M^{4} \times S^{1}$ can be expanded in Fourier harmonics in the coordinate $y$, in the case of the scalar field $\phi(x, y)$ the expansion being

$$
\phi(x, y)=L^{-\frac{1}{2}} \sum_{n} \phi^{(n)}(x) \exp \left(i \frac{2 \pi n y}{L}\right) .
$$

If one substitutes (2.2) into the five-dimensional equation of motion, one gets the corresponding equations for the modes $\phi^{(n)}$

$$
\left(\partial_{\mu} \partial^{\mu}-m_{n}^{2}\right) \phi^{(n)}=0, \quad m_{n}^{2}=m^{2}+\frac{4 \pi^{2} n^{2}}{L^{2}} .
$$

Thus, the mass spectrum of four-dimensional fields is defined by the eigenvalues of the mass operator, which in this case is just $\partial_{4} \partial^{4}$. Such a mass spectrum is a characteristic feature of the theories with extra dimensions, the form of the mass operator being defined by the geometry of the space of extra dimensions.

As we noted, O. Klein assumed the size $L$ of the extra dimension to be of the order of the Planck length. Within the framework of the mathematical apparatus of the modern quantum field 
theory this assumption leads to the conclusion that the observed fields in the Kaluza-Klein theory (i.e., the fields of the Standard Model) should be the so called zero modes, i.e. should not depend on the coordinates of extra dimensions. Moreover, for each observed field there should exist a tower of fields with masses of the order of the Planck mass $M_{P l} \sim 1 / l_{P l}$, which cannot be observed at the energies, which are available for experiments nowadays.

Nevertheless, even in this case there remain relations between the multidimensional and the four-dimensional theories, which can manifest themselves as certain symmetries of the reduced four-dimensional theories and the values of their coupling constants. In particular, an important point is the relation between the Planck mass in four dimensions $M_{P l}$ and the Planck mass $M$ in multidimensional space-time

$$
M_{P l}^{2}=M^{3} L,
$$

which can be obtained for the zero modes of gravitational field from action (2.1) in space-time $E=M^{4} \times S^{1}$ just by integrating it over the circle $S^{1}$.

\section{Large extra dimensions}

In the end of the sixties it was shown in paper [5] that a gravity theory invariant under the action of a Lie group $G$ in space-time $E=M^{4} \times G$ renders a gauge field in the four-dimensional space-time $M^{4}$ with the gauge group $G$. In other words, a non-abelian generalization of Kaluza-Klein theory was found, which led to a revival of the interest in theories with extra dimensions. There were numerous attempts to construct the Standard Model along these lines starting from a multidimensional theory. Since the spinor fields could not be derived from the metric of the multidimensional space-time, such fields and also gauge and scalar fields were introduced into the multidimensional theory from the very start. The interpretation of a theory in a space-time with extra dimensions in terms of a four-dimensional one was given then the name of dimensional reduction. To explain the factorized structure of the multidimensional space-time dynamically as a result of the interaction of gravity with gauge and scalar fields, the idea of spontaneous compactification was put forward. The attempts to construct the Standard Model in this approach showed that large extra dimensions were needed [6], which was obviously in contradiction with their unobservability.

In 1983 Rubakov and Shaposhnikov found a solution to this problem and put forward a new scenario for Kaluza-Klein theories based on the idea of localization of states [D]. They considered a real Higgs field in 5-dimensional space-time with the Lagrangian

$$
\mathscr{L}=-\frac{1}{2} \partial_{M} \phi \partial^{M} \phi-\frac{\lambda}{4}\left(\phi^{2}-\frac{m^{2}}{\lambda}\right)^{2} .
$$

The Higgs vacua of the system are $\phi^{0}= \pm \frac{m}{\sqrt{\lambda}}$ and an exact solution connecting these vacua is

$$
\phi^{c l}(y)=\frac{m}{\sqrt{\lambda}} \operatorname{th}\left(\frac{m y}{\sqrt{2}}\right) .
$$

The energy density of the solution is localized in the vicinity of the 3-dimensional hypersurface $y=0$ and has a width of the order $1 / \mathrm{m}$. Such an object is called a thick domain wall and serves as a potential well for the fluctuations of the field $\phi(x)$. 
It was shown that there are only two modes of the fluctuations that are localized on the domain wall. Moreover, interaction of these modes cannot produce unlocalized modes, if the center of mass energy is less than $\sqrt{6} m$. Thus, for sufficiently small energies the theory describes a 4-dimensional world, although the whole world is, in fact, 5-dimensional.

This model suggests a possibility for extra dimensions to be unobservable, if the fields of the Standard Model are localized by a certain mechanism on a three-dimensional hypersurface in multidimensional space-time. The corresponding localization mechanism turned out to be rather simple for spinors, but a plausible mechanism for gauge fields has not been found yet.

Rubakov and Shaposhnikov also proposed the following ansatz for multidimensional metric, which is compatible with this hypothesis [8]:

$$
d s^{2}=e^{-2 \sigma\left(y^{i}\right)} g_{\mu v} d x^{\mu} d x^{v}+g_{i k} d x^{i} d x^{k},
$$

$\left\{y^{i}\right\}$ being the coordinates of extra dimensions.

If the thickness of the domain wall goes to zero, it turns into a three-dimensional hypersurface in the multidimensional space, on which the field of the Standard Model are presumably trapped; such an object is called a membrane, or just a "brane". There are indications that this scenario can be implemented in the theory of superstrings.

It is clear that within the framework of this scenario extra dimensions may be much larger, than the Planck length (or even infinite). In paper [9] a single brane without tension (i.e. energy density) in a space-time with an arbitrary number of compact extra dimensions was considered. In this case relation (2.3) between the multidimensional Planck mass and the four-dimensional one, which for $d$ extra dimensions looks like

$$
M_{P l}^{2}=M^{d+2} L^{d},
$$

yields that the Planck mass in multidimensional space (in the bulk) $M$ may be much less than the Planck mass in four dimensions $M_{P l}$, if the volume of the space of extra dimensions $L^{d}$ is large. In other words, gravity in multidimensional space-time becomes "strong" not at the energies of the order of $10^{19} \mathrm{GeV}$, but at much lower energies, maybe of the order of $1-10 \mathrm{TeV}$. Thus, the scenario provides a solution to the hierarchy problem: it gives a strong gravity in the multidimensional space-time and a weak gravity on the brane. The effects due to the interaction of this "strong" gravity with the fields of the Standard Model could be observable already at the energies, which are available at the existing colliders.

A flaw of this approach is the approximation of the zero brane tension, which is rather too rough; it turns out that the proper gravitational field of the brane cannot be taken into account perturbatively at all. Moreover, for the equations of Einstein gravity in spaceÚtime with compact extra dimensions to be consistent, there should exist at least two branes with tension, and the number of extra dimensions can be either one or two, because otherwise branes turn into black holes.

In paper [10] the first exact solution for two branes interacting with gravity in five-dimensional space-time was found, which allows one to estimate the influence of the proper gravitational field of the brane on these results. We would like to note that the background metric of this solution is exactly of form (3.1). Below we will discuss in more detail the five-dimensional model, which is based in this solution. 


\section{The Randall-Sundrum model}

This model is called the Randall-Sundrum model and is similar to the original Kaluza-Klein theory in the sense that it is a gravity theory in 5-dimensional space-time $E=M^{4} \times S^{1}$ with coordinates $X^{M}=\left(x^{\mu}, y\right)$, the circle $S^{1}$ now being of circumference $2 L$. But unlike the original Kaluza-Klein theory the gravity interacts with two 3-branes. Thus, the action of the system is

$$
S=\frac{1}{16 \pi \hat{G}} \int d^{4} x \int_{-L}^{L} d y(\hat{R}-\Lambda) \sqrt{-g}-\lambda_{1} \int_{y=0} \sqrt{-\tilde{g}} d^{4} x-\lambda_{2} \int_{y=L} \sqrt{-\tilde{g}} d^{4} x
$$

where $\Lambda$ is the cosmological constant in 5-dimensional space-time, $\tilde{g}_{\mu \nu}$ is the metric induced on the branes, $\tilde{g}=\operatorname{det} \tilde{g}_{\mu \nu}$, and the subscripts 1,2 distinguish the branes. Moreover, the 5-metric $g$ must be invariant under the reflection $\left(x^{\mu}, y\right) \rightarrow\left(x^{\mu},-y\right)$, which is equivalent to identifying the points $\left(x^{\mu}, y\right)$ and $\left(x^{\mu},-y\right)$ of $S^{1}$ and reducing it to the orbifold $S^{1} / Z_{2}$.

If ansatz (3.1) for the metric, which in the case of one extra dimension looks like

$$
d s^{2}=e^{-2 \sigma(y)} \eta_{\mu \nu} d x^{\mu} d x^{v}+d y^{2} \equiv \gamma_{M N}(y) d x^{M} d x^{N},
$$

is substituted into Einstein equations following from action (4.1), they reduce to

$$
\begin{aligned}
\left(\frac{d \sigma}{d y}\right)^{2} & =-\frac{\Lambda}{12} \\
6 \frac{d^{2} \sigma}{d y^{2}} & =16 \pi \hat{G}\left(\lambda_{1} \delta(y)+\lambda_{2} \delta(y-L)\right) .
\end{aligned}
$$

The solution to these equation with the orbifold symmetry is

$$
\begin{aligned}
\sigma(y) & =k|y|+c, \quad \Lambda=-12 k^{2} \\
\lambda_{1} & =-\lambda_{2}=\frac{3 k}{4 \pi \hat{G}},
\end{aligned}
$$

where $k$ is a new parameter of the dimension of mass, which can be interpreted as the inverse effective thickness of the branes, and $\mathrm{c}$ is an integrations constant. The choice of this constant is equivalent to a choice of the coordinates $\left\{x_{\mu}\right\}$ on the branes, and we will keep it arbitrary for the moment.

Two remarks are in order. First, the space $E$ with metric (4.2) and $\sigma$ given by solution (4.3) is a piece of five-dimensional anti-de Sitter space in horospherical coordinates [11]. Second, we see that brane 1 has a positive energy density, whereas brane 2 has a negative one.

Metric (4.2) with $\sigma$ given by equation (4.3) is taken to be the vacuum of the theory, and the physical fields are fluctuations $h_{M N}(x, y)$ above this vacuum, which are treated perturbatively:

$$
g_{M N}(x, y)=\gamma_{M N}(y)+\hat{\kappa} h_{M N}(x, y)
$$

where $\hat{\kappa}=\sqrt{16 \pi \hat{G}}$.

If we substitute this representation into action (4.1) and keep the terms of zero order in $\hat{\kappa}$, we get a free action for the physical degrees of freedom of the model. The corresponding Lagrangian looks like 


$$
\begin{aligned}
\mathscr{L} / \sqrt{-\gamma} & =-\frac{1}{4}\left(\nabla_{R} h_{M N} \nabla^{R} h^{M N}-\nabla_{R} h \nabla^{R} h+2 \nabla_{M} h^{M N} \nabla_{N} h-\right. \\
& \left.-2 \nabla_{M} h^{M N} \nabla^{R} h_{R N}\right)+\frac{k^{2}}{2}\left(h_{M N} h^{M N}+h h\right)+ \\
& +\left[-2 k h_{M N} h^{M N}+k h \tilde{h}-k h_{M v} h^{M v}+3 k\left(h_{\mu v} h^{\mu v}-\frac{1}{2} \tilde{h} \tilde{h}\right)\right] \tilde{\delta},
\end{aligned}
$$

where $\gamma=\operatorname{det} \gamma_{M N}, h=\gamma^{M N} h_{M N}, \nabla_{M}$ stands for the covariant derivative with respect to metric $\gamma_{M N}$, and $\tilde{\delta}=\delta(y)-\delta(y-L)$.

It is a common knowledge that linearized gravity is a gauge theory, and to isolate the physical degrees of freedom we have to study the gauge invariance of this Lagrangian.

On can check that the Lagrangian is invariant under the gauge transformations

$$
\begin{aligned}
h_{\mu v}^{(\prime)}(x, y) & =h_{\mu v}(x, y)-\left(\partial_{\mu} \xi_{v}+\partial_{\nu} \xi_{\mu}-2 \gamma_{\mu v} \partial_{4} \sigma \xi_{4}\right) \\
h_{\mu 4}^{(\prime)}(x, y) & =h_{\mu 4}(x, y)-\left(\partial_{\mu} \xi_{4}+\partial_{4} \xi_{\mu}+2 \partial_{4} \sigma \xi_{\mu}\right) \\
h_{44}^{(\prime)}(x, y) & =h_{44}(x, y)-2 \partial_{4} \xi_{4},
\end{aligned}
$$

where $\partial_{4} \equiv \frac{\partial}{\partial y}$ and the gauge functions $\xi_{M N}(x, y)$ satisfy the orbifold symmetry conditions.

With the help of these transformations one can prove that there exist a gauge

$$
h_{\mu 4}=0, h_{44}=h_{44}(x) \equiv \phi(x),
$$

which we call the unitary gauge, because the main degrees of freedom, which include the tensor field $h_{\mu \nu}$ and the scalar filed $\phi(x)$, are already isolated. Nevertheless, this name is somewhat relative, because there remain gauge transformations, satisfying

$$
\partial_{4}\left(e^{2 \sigma} \xi_{\mu}\right)=0
$$

which are necessary to remove the gauge degrees of freedom of the massless graviton.

Equations of motion for different components of $h_{M N}(x, y)$ can be derived from (4.5) by the standard procedure and in the unitary gauge (4.7) look like:

1) $\mu v$-component

$$
\begin{aligned}
& \frac{1}{2}\left(\partial_{\rho} \partial^{\rho} h_{\mu v}-\partial_{\mu} \partial^{\rho} h_{\rho v}-\partial_{\nu} \partial^{\rho} h_{\rho \mu}+\frac{\partial^{2} h_{\mu v}}{\partial y^{2}}\right)-2 k^{2} h_{\mu v}+\frac{1}{2} \partial_{\mu} \partial_{v} \tilde{h}+ \\
+ & \frac{1}{2} \partial_{\mu} \partial_{\nu} \phi+\frac{1}{2} \gamma_{\mu v}\left(\partial^{\rho} \partial^{\sigma} h_{\rho \sigma}-\partial_{\rho} \partial^{\rho} \tilde{h}-\frac{\partial^{2} \tilde{h}}{\partial y^{2}}+4 \partial_{4} \sigma \partial_{4} \tilde{h}-\partial_{\rho} \partial^{\rho} \phi+12 k^{2} \phi\right) \\
+ & {\left[2 k h_{\mu v}-3 k \gamma_{\mu \nu} \phi\right] \tilde{\delta}=0, }
\end{aligned}
$$

2) $\mu 4$-component

$$
\partial_{4}\left(\partial_{\mu} \tilde{h}-\partial^{v} h_{\mu v}\right)+3 \partial_{4} \sigma \partial_{\mu} \phi=0
$$

which is a constraint,

3) 44-component

$$
\frac{1}{2}\left(\partial^{\mu} \partial^{v} h_{\mu v}-\partial_{\rho} \partial^{\rho} \tilde{h}\right)+\frac{3}{2} \partial_{4} \sigma \partial_{4} \tilde{h}+6 k^{2} \phi=0
$$


To explicitly isolate and to decouple the physical degrees of freedom we make a substitution

$$
h_{\mu v}=b_{\mu v}-\gamma_{\mu \nu}(y)(k|y|+s) \phi+\frac{1}{2 k^{2}}\left(\frac{1}{2}-k|y|-s+\frac{s}{2} e^{2 k|y|}\right) \partial_{\mu} \partial_{\nu} \phi, \quad s=\frac{k L}{\left(e^{2 k L}-1\right)} .
$$

It can be inferred from equations (4.9), (4.10), and (4.11) that the scalar field $\phi(x)$ is massless and the field $b_{\mu v}(x, y)$ can be made transverse-traceless by the remaining gauge transformations and satisfies the equation

$$
\frac{1}{2} e^{2 \sigma} \square b_{\mu v}+\frac{1}{2} \frac{\partial^{2} b_{\mu v}}{\partial y^{2}}-2 k^{2} b_{\mu v}+2 k b_{\mu v} \tilde{\delta}=0
$$

Thus, the fluctuations of the metric above the background of the Randall-Sundrum solution are described by the field $b_{\mu v}(x, y)$ of spin 2 and the massless scalar radion field $\phi(x)$.

Substitution (4.12) also diagonalizes Lagrangian (4.5), which gives that the canonically normalized radion field is

$$
\varphi=e^{-c} \sqrt{\frac{3 k L^{2}}{e^{2 k L}-1}} \phi .
$$

To understand the physical meaning of the radion field let us calculate the distance between the branes along a geodesic $x=$ const:

$$
l=\int_{0}^{L} \sqrt{d s^{2}} \sim \int_{0}^{L}\left(1+\frac{1}{2} \hat{\kappa} h_{44}\right) d y=L\left(1+\frac{1}{2} \hat{\kappa} \phi(x)\right) .
$$

This formula implies that the field $\phi(x)$ describes local variations of the brane separation, i.e. oscillations of the branes with respect to each other.

The tensor field $b_{\mu v}(x, y)$ can be expanded as

$$
b_{\mu v}(x, y)=\sum_{n} b_{\mu v}^{n}(x) \psi_{n}(y)
$$

where $b_{\mu \nu}^{n}(x)$ are four-dimensional fields with spin 2 and certain masses $m_{n}$. The latter and the wave functions of the modes $\psi_{n}(y)$ are defined by the equation

$$
\left[\frac{1}{2} \frac{d^{2}}{d y^{2}}+2 k(\delta(y)-\delta(y-L))-2 k^{2}\right] \psi_{n}(y)=\frac{m_{n}^{2}}{2} e^{2 \sigma} \psi_{n}(y)
$$

This equation can be solved exactly, the wave function of the zero mode being proportional to $\exp (-2 \sigma)$ and those of massive modes being expressible in terms of Bessel and Neumann functions.

Now we are in a position to find the relation between the 5-dimensional gravitational constant $\hat{G}$ and the 4-dimensional $G$, which will give us the relation between the Planck masses. To this end we have to calculate the curvature of the metric

$$
d s^{2}=e^{-2 \sigma(y)} \bar{g}_{\mu \nu} d x^{\mu} d x^{v}+d y^{2}
$$


with $\bar{g}_{\mu v}=\eta_{\mu v}+h_{\mu v}(x)$ and integrate it over the extra dimension. Since the wave function of the massless graviton is proportional to $\exp (-2 \sigma)$, this metric corresponds to four-dimensional gravity only and integrating it over the extra dimension gives an effective action for 4-dimensional gravity

$$
S_{e f f}=\frac{1}{16 \pi \hat{G}} \int_{-L}^{L} d y e^{-2(k|y|+c)} \int R(\bar{g}) \sqrt{-\bar{g}} d^{4} x=e^{-2 c} \frac{1-e^{-2 k L}}{16 \pi \hat{G} k} \int d^{4} x R(\bar{g}) \sqrt{-\bar{g}}
$$

which has to coincide with the Hilbert-Einstein action for the metric $\bar{g}_{\mu \nu}$. But since we have two branes, we have to realize, to which brane this metric corresponds.

If we are on the brane at $y=0$, the metric on the brane induced from metric (4.18) coincides with $\bar{g}_{\mu \nu}$ only if $c=0$. This leads to the following relation between the gravitational constants

$$
G_{1}=\frac{\hat{G} k}{1-e^{-2 k L}}
$$

which implies that relation (2.3) is modified to be

$$
M_{1}^{2}=M^{3} \frac{1-e^{-2 k L}}{k} .
$$

Thus, if we live on the brane at $y=0$, we have to put $M_{1}=M_{P l}$, and in this case relation 4.21) demands that the five-dimensional Planck mass $M$ and the parameter $k$ also should be of the order of $M_{P l}, \quad M \sim k \sim M_{P l}$. This means that for an observer on brane 1 the five-dimensional gravity is as weak, as the four-dimensional one.

If we are on the brane at $y=L$, the metric on the brane induced from metric (4.18) coincides with $\bar{g}_{\mu v}$ only if $c=-k L$. In this case formula (4.19) gives us the following relation between Newton's constant $G_{2}$ on brane 2 and the five-dimensional one

$$
G_{2}=\frac{\hat{G} k}{e^{2 k L}-1}
$$

and the corresponding relation between $M_{2}$ and the five-dimensional Planck mass M

$$
M_{2}^{2}=M^{3} \frac{e^{2 k L}-1}{k} .
$$

Obviously, both relations (4.21) - (4.23) reduce to (2.3) (with $L$ replaced by $2 L$ ) for $k \rightarrow 0$, i.e. when the effective thickness of the brane goes to infinity and the very notion of a brane is meaningless.

If our world is situated on the brane at $y=L$, we have to put $M_{2}=M_{P l}$. In this case relation (4.23) with $k L \sim 35$ gives that the five-dimensional Planck mass $M$ and the parameter $k$ may be of the order of $1 \mathrm{TeV}$. That is the hierarchy problem of the gravitational interaction is solved for an observer on brane 2: the energy scale of the five-dimensional gravity comes down to the electroweak energy scale, and the gravity on the brane becomes weak due to the exponential warp factor in relation (4.23). Thus, five-dimensional gravity in the Randall-Sundrum model looks different for observers on different branes.

In what follows we will assume that we live on the brane at $y=L$ and will consider the interaction of the physical fields with matter. This interaction can be derived from the standard 
action of matter in gravitational field and has the following form:

$$
\frac{\hat{\kappa}}{2} \int_{y=o} h^{\mu v}(x, 0) T_{\mu v}^{1} \sqrt{-\operatorname{det} \gamma_{\mu v}(0)} d x++\frac{\hat{\kappa}}{2} \int_{y=L} h^{\mu v}(x, L) T_{\mu v}^{2} \sqrt{-\operatorname{det} \gamma_{\mu v}(L)} d x,
$$

where $T_{\mu \nu}^{1}$ and $T_{\mu \nu}^{2}$ are energy-momentum tensors of matter on brane 1 and brane 2 respectively:

$$
T_{\mu \nu}^{1,2}=2 \frac{\delta L^{1,2}}{\delta \gamma^{\mu \nu}}-\gamma_{\mu \nu}^{1,2} L^{1,2}
$$

On the brane at $y=L$ the explicit form of this interaction is

$$
S_{i n t}=\frac{1}{2} \int_{y=L}\left(\kappa_{2} b_{\mu v}^{0}(x) T^{\mu v}+\kappa_{1} \sum_{n=1}^{\infty} \frac{\psi_{n}(L) e^{-k L}}{N_{0}} b_{\mu v}^{n}(x) T^{\mu v}-\frac{\kappa_{1}}{\sqrt{3}} \varphi T_{\mu}^{\mu}\right) d x
$$

where

$$
\kappa_{1}=\hat{\kappa} \sqrt{\frac{k}{1-e^{-2 k L}}}, \quad \kappa_{2}=\kappa_{1} e^{-k L}=\hat{\kappa} \sqrt{\frac{k}{e^{2 k L}-1}},
$$

and the factor $\frac{\psi_{n}(L) e^{-k L}}{N_{0}}$ in front of $b_{\mu \nu}^{n}(x)$ turns out to be of the order of unity for small $n$.

With the help of this Lagrangian one can calculate the effects due to the interaction of the five-dimensional gravity with matter on brane 2. In particular, one gets for Newton's law

$$
V=-G_{2}\left(1+\frac{e^{2 k L}}{3}\right) \frac{m}{r}
$$

The second term in the brackets in this formula is the contribution of the radion field, which is $e^{2 k L} / 3 \sim 10^{32}$ times larger than the contribution of the massless graviton. Thus, a scalar gravity is realized on brane 2, which is phenomenologically unacceptable. A way to cure this problem without violating the solution of the hierarchy problem is to give a mass to the radion. It turns out that this is equivalent to stabilizing the distance between the branes.

\section{Stabilized Randall-Sundrum model}

Mechanisms for stabilizing the Randall-Sundrum model were put forward in papers [12, 13]. Both of them employ a five-dimensional scalar field to stabilize the inter-brane distance, but the mechanism of paper [13] seems to be preferable because it is based on an exact solution for gravity interacting with two branes and a scalar field in five-dimensional space-time. The physical degrees of models stabilized by this mechanism were isolated in paper [14]. They are tensor fields $b_{\mu}^{n}(x), n=0,1, \cdots$ with masses $m_{n}\left(m_{0}=0\right)$ and wave functions in the space of extra dimension $\left.\psi_{n}(y)\right)$, and scalar fields $\varphi_{n}(x), n=1,2, \cdots$ with masses $\mu_{n}$ and wave functions in the space of extra dimension $g_{n}(y)$. Their interaction with the SM fields is described by the Lagrangian

$$
S_{i n t}=\frac{\hat{\kappa}}{2} \int_{y=L}\left(\psi_{0}(L) b_{\mu v}^{0}(x) T^{\mu v}+\sum_{n=1}^{\infty} \psi_{n}(L) b_{\mu v}^{n}(x) T^{\mu v}-\frac{1}{2} \sum_{n=1}^{\infty} g_{n}(L) \varphi_{n}(x) T_{\mu}^{\mu},\right) d x .
$$


$T^{\mu v}$ being the energy-momentum tensor of the SM fields.

The wave function of the zero mode $\psi_{0}(L)$ again gives us relation (4.23) between the fiveand four-dimensional Planck masses. The coupling constants to matter of the massive tensor fields remain essentially the same as in the unstabilized model, i.e. of the order of $1 \mathrm{TeV}^{-1}$. The couplings of the scalar fields are also defined by their wave functions and are essentially of the same order.

The present-day results from the Tevatron [15] indicate that we do not see the resonances, corresponding to these tensor and scalar modes. We can assume that we have not yet reached the energies necessary for their production. In this case the interactions due to exchange of these tensor and scalar modes can be very well approximated by contact interactions of a very special form:

$$
\begin{aligned}
L_{e f f} & =\frac{1.82}{\Lambda_{\pi}^{2} m_{1}^{2}} T^{\mu v} \tilde{\Delta}_{\mu v, \rho \sigma} T^{\rho \sigma}, \\
\tilde{\Delta}_{\mu v, \rho \sigma} & =\frac{1}{2} \eta_{\mu \rho} \eta_{v \sigma}+\frac{1}{2} \eta_{\mu \sigma} \eta_{v \rho}-\left(\frac{1}{3}-\frac{\delta}{2}\right) \eta_{\mu v} \eta_{\rho \sigma},
\end{aligned}
$$

where $m_{1}$ and $\Lambda_{\pi}$ are the mass and the coupling constant of the first tensor mode and the constant $\delta$ describes the contribution of the scalar modes. For example, for $M=2 \mathrm{TeV}, k=1 \mathrm{TeV}, k L=35$ and the mass of the first scalar mode of the order of $2 \mathrm{TeV}$ these parameters turn out to be $\Lambda_{\pi} \simeq$ $8 T e V, m_{1} \simeq 3.83 \mathrm{TeV}, \delta \simeq 0.7$.

\section{Models with more than one extra dimension}

When one considers the Randall-Sundrum model, a natural question arises: is it possible to construct such models with more than one extra dimension? The answer to this question essentially depends on the extension of gravity theory to the multidimensional space-time. For example, for the extension given by formula (2.1) it is impossible to construct brane world models with more than two extra dimensions, because in this extension we still have the Einstein gravity in multidimensional space-time, and branes with more than two extra dimensions turn into black holes. A solution for two branes in space-time with two extra dimenions with a metric corresponding to a factorizeable geometry was found in [16].

A possible solution to the problem can be in passing to Lovelock gravity [17] in the multidimensional space-time instead of the Eistein one. The Lovelock Lagrangian for gravity theory in $d$-dimensional space-time looks like:

$$
16 \pi G_{d} \mathscr{L}_{L}=\sum_{0 \leq p \leq(d+1) / 2} \alpha_{p} \lambda^{2(p-1)} \mathscr{L}_{(p)},
$$

where

$$
\mathscr{L}_{(p)}=\frac{1}{2^{p}} \delta_{J_{1} J_{2} \cdots J_{2 p}}^{I_{1} I_{2} \cdots I_{2 p}} R_{I_{1} I_{2}}^{J_{1} J_{2}} \cdots R^{J_{2 p-1} J_{2 p}} I_{2 p-1} I_{2 p}
$$

$\delta_{J_{1} J_{2} \cdots J_{2 p}}^{I_{1} I_{2} \cdots I_{2}}$ is a generalized Kronecker delta, $\alpha_{p}$ is a dimensionless parameter and $\lambda$ is a parameter of the dimension of mass.

In particular, we have for the first three terms in the sum:

$$
\mathscr{L}_{(0)}=1, \quad \mathscr{L}_{(1)}=R, \quad \mathscr{L}_{(2)}=R^{P R}{ }_{M N} R_{P R}^{M N}-4 R_{M N} R^{M N}+R^{2} .
$$


Thus, $\mathscr{L}_{(0)}$ is the cosmological constant, $\mathscr{L}_{(1)}$ is the standard Lagrangian of the Einstein gravity, and $\mathscr{L}_{(2)}$ is the Gauss-Bonnet term, which is often used in multidimensional extensions of gravity.

In paper [18] it was argued that passing to Lovelock gravity could allow one to construct brane world models with more than two extra dimensions.

\section{Processes with Kaluza-Klein gravitons}

In this section we will study processes with Kaluza-Klein gravitons within the stabilized Randall-Sundrum model. In the first approximation in the SM coupling constants the effective interaction Lagrangian (5.2) includes a sum of various 4-particle effective operators (not only 4fermion, but also 2-fermion-2-boson and 4-boson), which are invariant with respect to the SM gauge group and lead to a well defined phenomenology. Various processes due to this Lagrangian were studies in paper [19].

Below we show the results of numerical studies of Drell-Yan processes, because they are most sensitive to new physics. These analytical and numerical calculations, including the Monte-Carlo simulation of the SM background in a Gedankenexperiment for the LHC, were carried out with the help of the CompHEP package [20], realized on the basis of the package for symbolic calculations FORM [22]. The Feynmann rules following from effective Lagrangian (5.2) were incorporated into this programme, which allows one to use this code for event generation and then for data analysis in a real experiment.

In figure 1 dilepton invariant mass distributions are plotted for different values of the coupling parameter $0.91 / \Lambda_{\pi}^{2} m_{1}^{2}$ for the Tevatron. In figures 2,3 such distributions are plotted for $0.91 / \Lambda_{\pi}^{2} m_{1}^{2} \times T e V^{4}=0.66$ for the Tevatron and for $0.91 / \Lambda_{\pi}^{2} m_{1}^{2} \times T e V^{4}=0.0014$ for the LHC.

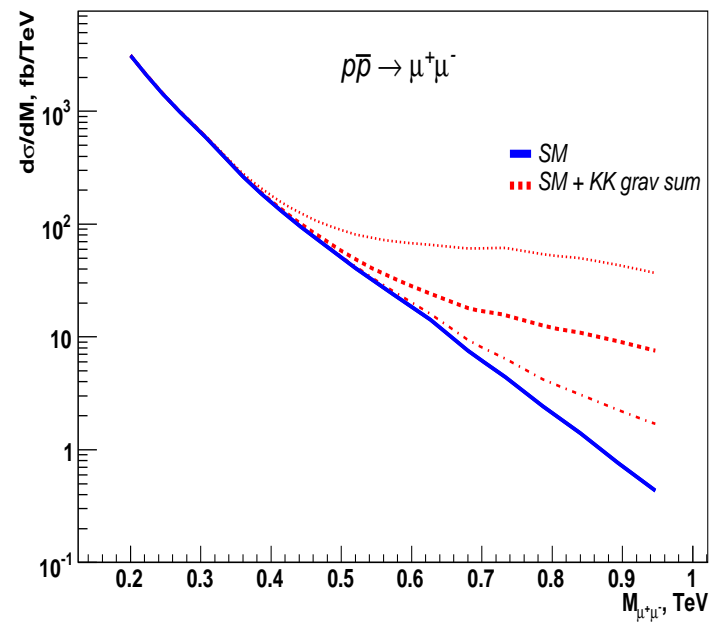

Figure 1: Dilepton invariant mass distribution for parameters $\frac{0.91}{\Lambda_{\pi}^{2} m_{1}^{2}} \times T e V^{4}=0.66$ (dash-dotted line), 1.82 (dashed line), 4 (dotted line) for the Tevatron

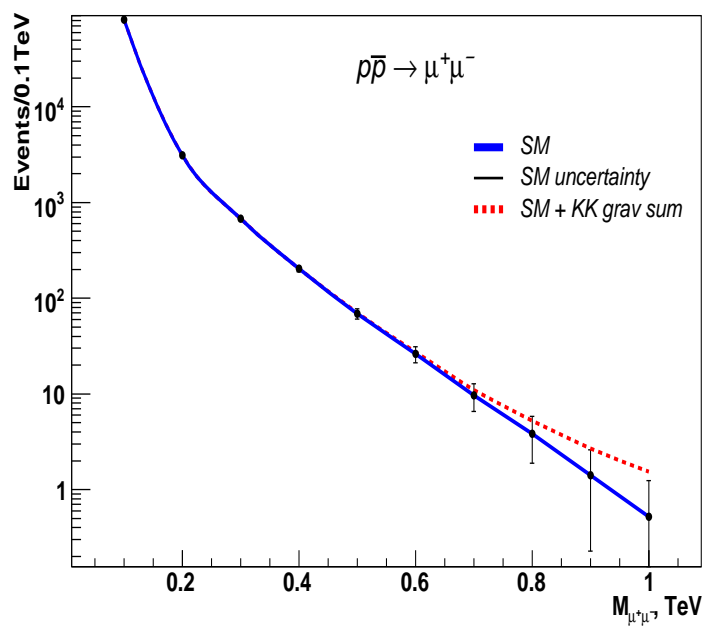

Figure 2: Dilepton invariant mass distribution for 95\% CL parameter $\frac{0.91}{\Lambda_{\pi}^{2} m_{1}^{2}} \times T e V^{4}=0.66$ for the Tevatron $\left(L=10 \mathrm{fb}^{-1}\right)$ 
The error bars in these figures are not usual error bars, but take into account both systematic errors (detector resolution, QCD uncertainties, errors in electroweak parameters and luminosity) and the statistical uncertainties of the dilepton invariant mass distribution and define the values of the coupling parameter, for which the contact interaction cannot be observed at the Tevatron and the LHC. For the largest luminosities, they are

$$
\text { Tevatron }\left(10 \mathrm{fb}^{-1}\right): \frac{0.91}{\Lambda_{\pi}^{2} m_{1}^{2}} \times T e V^{4}<0.66, \quad \operatorname{LHC}\left(100 \mathrm{fb}^{-1}\right): \frac{0.91}{\Lambda_{\pi}^{2} m_{1}^{2}} \times T e V^{4}<0.0014 .
$$

These constraints can be used to estimate the lowest value of parameter $\Lambda_{\pi}$, for which the effects of the contact interaction cannot be resolved, from the demand that the width of the resonance is less than its mass: $\Gamma_{1}<m_{1} / \xi$, where $\xi$ is a number, $\xi>1$. Utilizing (7.1) and the expression for the total graviton width [19], we find the following unobservability range for the parameter $\Lambda_{\pi}$ :

$$
\text { Tevatron : } \Lambda_{\pi}>0.61 \cdot \xi^{1 / 4} \mathrm{TeV}, \quad \mathrm{LHC}: \Lambda_{\pi}>2.82 \cdot \xi^{1 / 4} \mathrm{TeV}, \quad \xi>1 .
$$

Next we consider the case, where the mass of the first KK resonance is within or close to the accessible energy range. Here the approximation of the interaction due to the exchange of the first KK resonance by a contact interaction fails, and we have to take it into account exactly. The contribution of all the KK modes above it can still be described by effective Lagrangian (5.2) with the coupling parameter $0.41 / \Lambda_{\pi}^{2} m_{1}^{2}$, remaining after dropping the contribution of the first resonance to the sum.

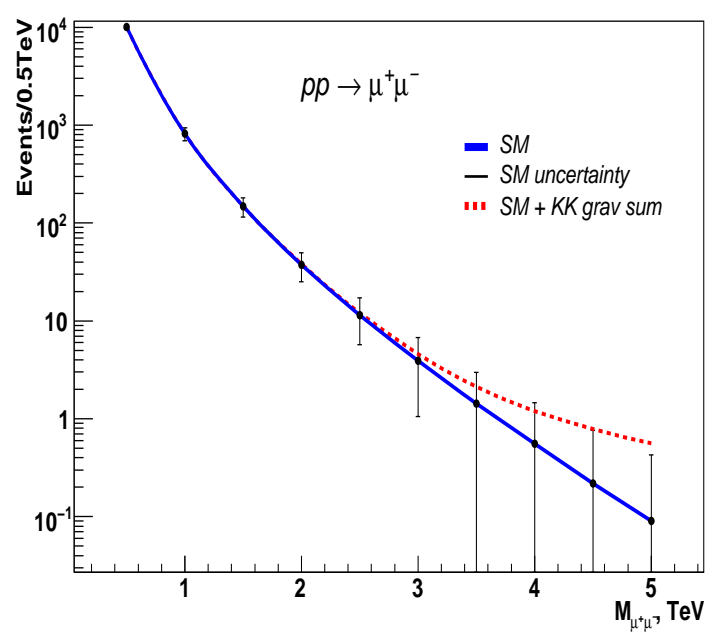

Figure 3: Dilepton invariant mass distribution for 95\% CL parameter $\frac{0.91}{\Lambda_{\pi}^{2} m_{1}^{2}} \times T e V^{4}=0.0014$ for the $\mathrm{LHC}$ $\left(L=100 \mathrm{fb}^{-1}\right)$

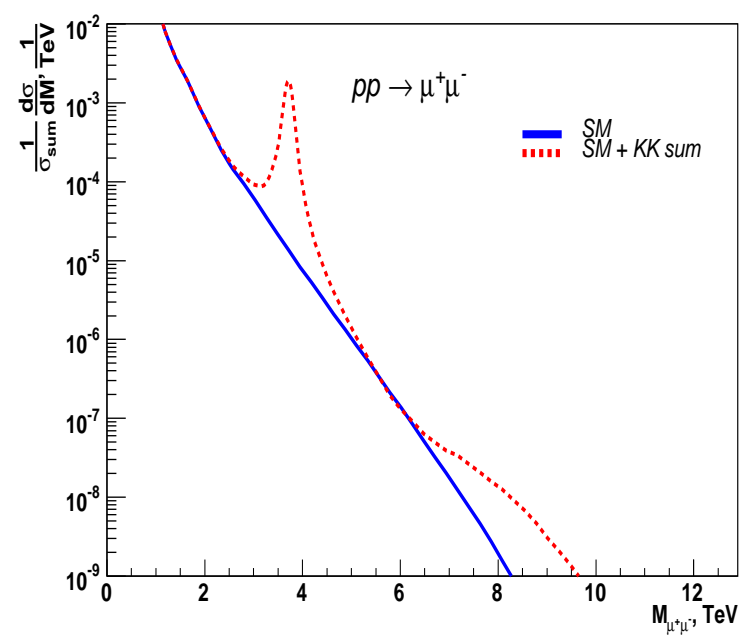

Figure 4: Dilepton invariant mass distribution from the SM (solid line) and from the SM plus sum of KK modes including the first $\mathrm{KK}$ resonance with $M_{\text {res }}=$ $3.83 \mathrm{TeV}, \Gamma_{\text {res }}=0.08 \mathrm{TeV}, \Lambda_{\pi}=8 \mathrm{TeV}($ dashed line $)$ for the $\mathrm{LHC}$

To illustrate the modification of distributions due to the contributions of the towers of KK gravitons we carried out the calculations for two sets of parameters chosen so that for one set the 
first KK resonance lies within the energy range of direct observation and for the other one beyond it. The first set includes the already mentioned values $m_{1}=3.83 \mathrm{TeV}, \Lambda_{\pi}=8 \mathrm{TeV}$ and the width of the first resonance $\Gamma_{1}=0.08 \mathrm{TeV}$. In the context of the RS1 model such a resonance (figure 4) lies close to the boundary of direct observation expected for the LHC. The mass of the first KK resonance in the second set of parameters $\left(m_{1}=10 \mathrm{TeV}, \Lambda_{\pi}=14 \mathrm{TeV}, \Gamma_{1}=0.5 \mathrm{TeV}\right)$ is close to the maximal collider energy and it is not directly observable.

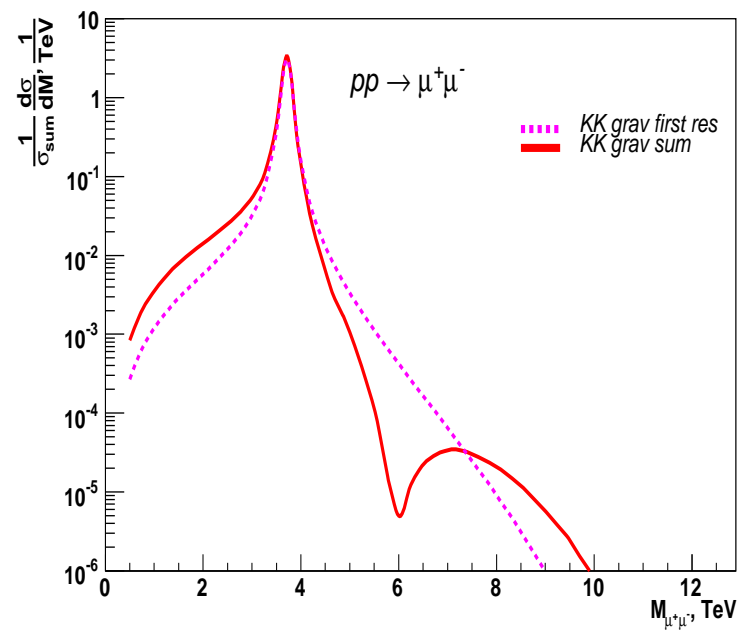

Figure 5: The normalized dilepton invariant mass distribution from the first $\mathrm{KK}$ resonance plus the sum of KK tower states starting from the second mode (solid line) and from the first KK resonance only (dashed line) for $M_{\text {res }}=3.83 \mathrm{TeV}, \Gamma_{\text {res }}=$ $0.08 \mathrm{TeV}, \Lambda_{\pi}=8 \mathrm{TeV}$ for the LHC

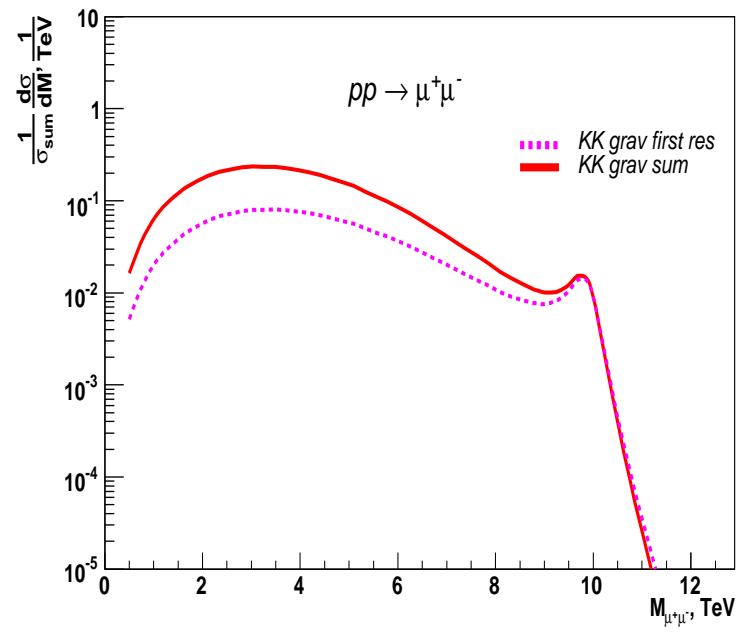

Figure 6: The normalized dilepton invariant mass distribution from the sum of KK tower states starting from the first mode (solid line) and from the first KK resonance only (dashed line) for $M_{\text {res }}=10 \mathrm{TeV}, \Gamma_{\text {res }}=$ $0.5 \mathrm{TeV}, \Lambda_{\pi}=14 \mathrm{TeV}$ for the LHC

In figure 5 and figure 6 one can see that the contribution of the KK tower increases the production cross-section more than three times for invariant masses below the first KK resonance mass. The situation is quite different for invariant masses above the first resonance mass, where, on a par with the resonance peak, there appears a dip due to the destructive interference between the contribution of the first KK resonance and the contribution of the rest of the KK tower. The local minimum is at $M_{\min } \approx 1.5 \mathrm{~m}_{1}$. The cross-section growth after this minimum is strongly suppressed by the parton structure functions, which leads to an extra hump in the distribution in the invariant mass. However, figure 6 shows that an experimental observation of this hump against the SM background is rather unlikely.

Similar interference effects take place, when one considers KK towers of the SM fields.

\section{Universal Extra Dimensions and processes with the excitations of the SM gauge bosons}

If the SM gauge bosons can propagate in the bulk, there also arise KK towers of their excitations, which may produce similar effects. First we consider the simpler case of the $W$ boson and 
its KK tower. The coupling constants of its excitations and their masses essentially depend on the fundamental parameters of a stabilized brane-world model, which is also true for the excitations of other particles to be discussed below. In particular, in paper [19] the masses of the graviton excitations were explicitly calculated in terms of the fundamental parameters, which turned out to be a rather complicated task.

Under these assumptions we will study the processes $p p \rightarrow t \bar{b}+X$ at the LHC. It occurs due to the weak process $u \bar{d} \rightarrow t \bar{b}$, which is mediated by the $W$ boson and its KK tower. In our approximation the amplitude of the process can be represented by the diagrams

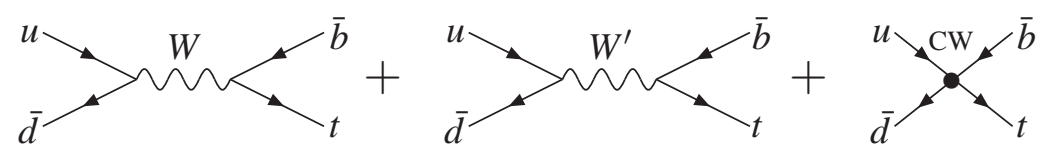

The contact term CW is, in fact, Fermi's interaction with the coupling constant $g_{1}^{2} /\left(2 M_{W^{\prime}-\text { sum }}^{2}\right)$. Explicit calculations in UED models with flat extra dimension and in certain stabilized braneworld models show that this mass is just a little larger than that of $W^{\prime}$. Here we will take the value $M_{W^{\prime} \_ \text {sum }}=1.4 M_{W^{\prime}}$, previously used in [21], as the effective mass of the KK tower above $W^{\prime}$ and will apply the same relation to the excitations of the other gauge bosons.

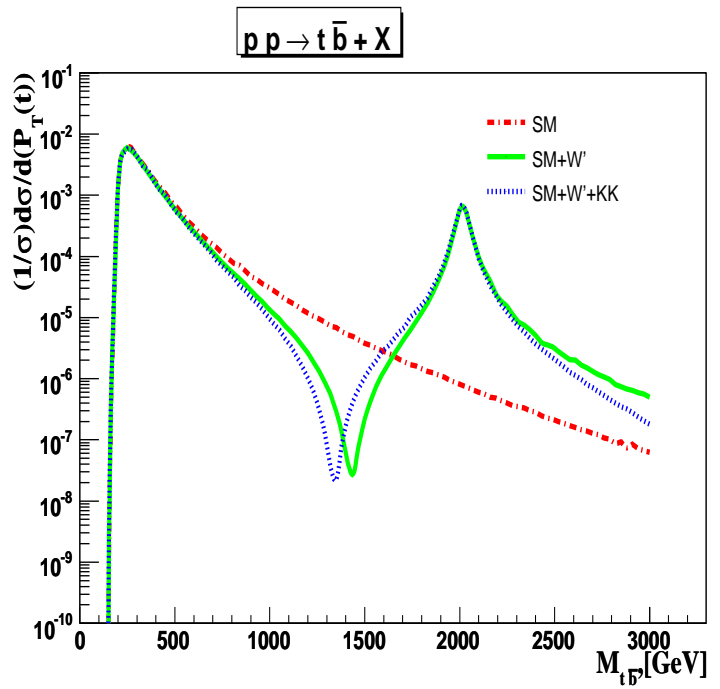

Figure 7: Distribution in the invariant mass of $t \bar{b}$ pair for $M_{W^{\prime}}=2 \mathrm{TeV}$ for the LHC. Dash-dotted line corresponds to the SM, solid line corresponds to the sum $\mathrm{SM}+\mathrm{W}^{\prime}$, dashed line corresponds to the sum $\mathrm{SM}+\mathrm{W}^{\prime}+\mathrm{KK}$.

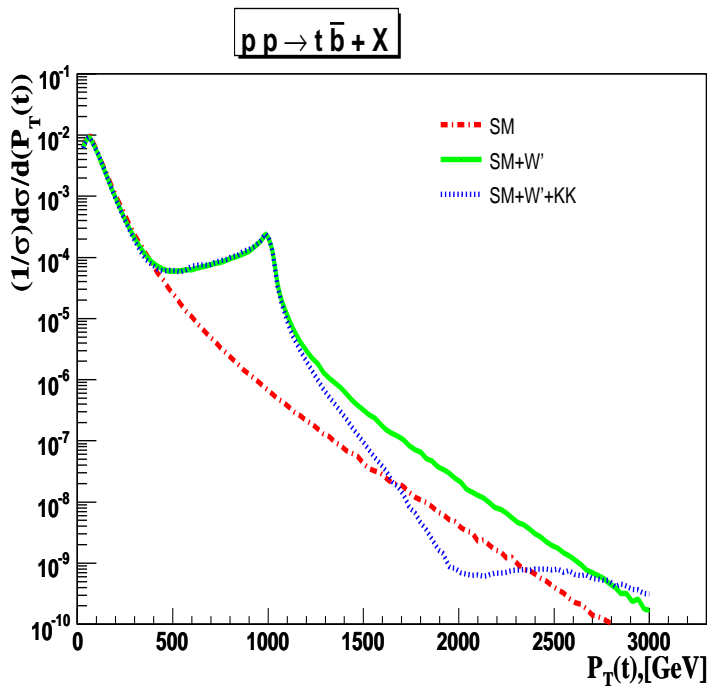

Figure 8: Top quark $p_{t}$ distribution for $M_{W^{\prime}}=2 \mathrm{TeV}$ for the LHC. Dash-dotted line corresponds to the SM, solid line corresponds to the sum $\mathrm{SM}+\mathrm{W}^{\prime}$, dashed line corresponds to the sum $\mathrm{SM}+\mathrm{W}^{\prime}+\mathrm{KK}$.

The cross-sections of this process can be obtained by calculating the corresponding partonic cross-sections and integrating them with the parton distribution functions, and in so doing we neglect the light quark masses. The results of these calculations are presented in figures 7 and 8 and show that the interference with the contribution of the rest of the KK tower changes the 
curves significantly.

Here we will also consider processes with the excitations of the neutral SM gauge bosons. Examples of processes mediated by $Z^{\prime}, \gamma^{\prime}$ and the corresponding KK towers are the $t \bar{t}$ production process and the Drell-Yan process $p p \rightarrow \mu^{+} \mu^{-}+X$. The effective Lagrangians for the KK towers of $\gamma$ and $Z$ have the same form as the one for the $W$ boson with the weak charged current $J^{+\mu}$ replaced by the electromagnetic current and the weak neutral current respectively. In this case the process $u \bar{u} \rightarrow \mu^{+} \mu^{-}$is described in our approximation by the following diagrams:

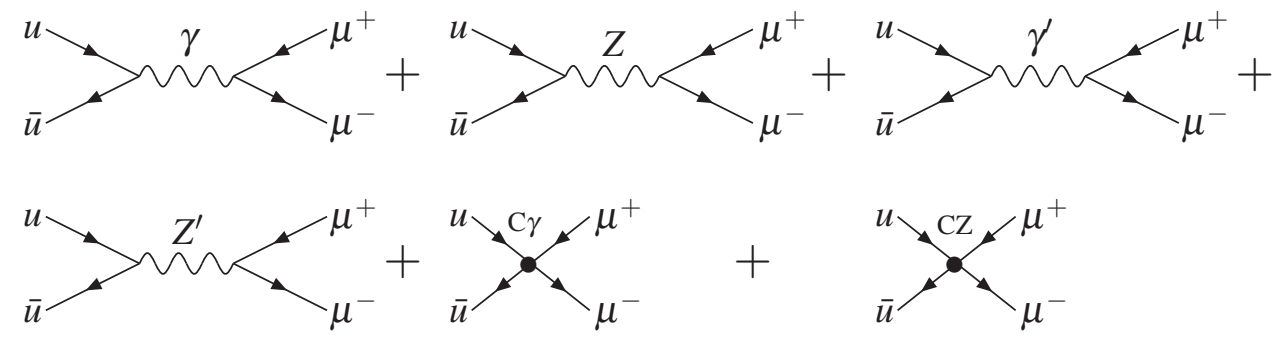

The diagrams for the process of $t \bar{t}$-production can be obtained by replacing the $\mu^{+} \mu^{-}$pair by $t \bar{t}$ pair.

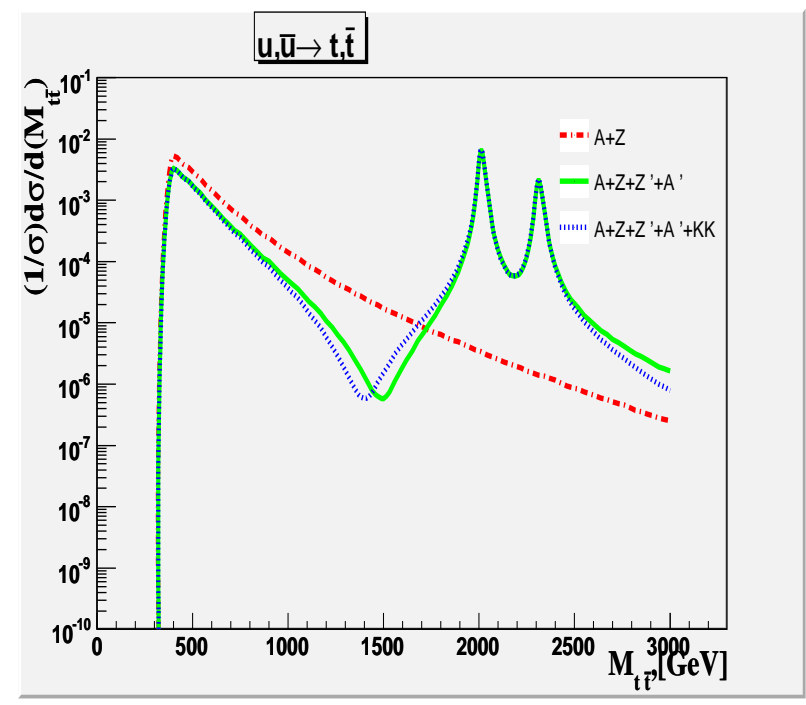

Figure 9: Distribution in the invariant mass of $t \bar{t}$ pair for $M_{\gamma^{\prime}}=2 \mathrm{TeV}, \quad M_{Z^{\prime}}=2.3 \mathrm{TeV}$ for the LHC. Dashdotted line corresponds to the SM, solid line corresponds to the sum $\mathrm{SM}+\mathrm{A}^{\prime}+\mathrm{Z}^{\prime}$, dashed line corresponds to the sum $\mathrm{SM}+\mathrm{A}^{\prime}+\mathrm{Z}^{\prime}+\mathrm{KK}$.

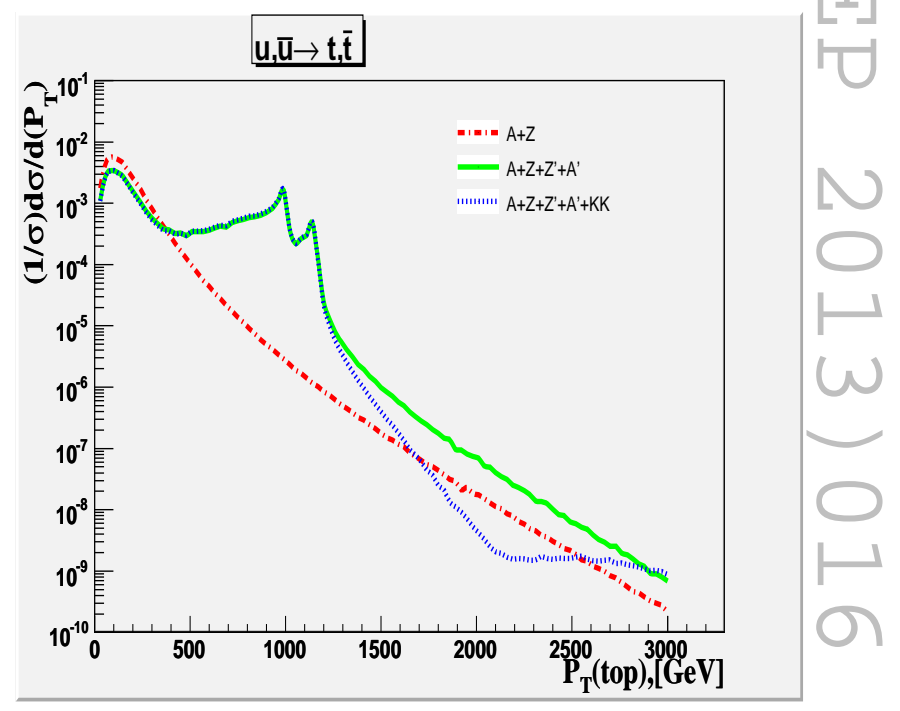

Figure 10: Top quark $p_{t}$ distribution for $M_{\gamma^{\prime}}=$ $2 \mathrm{TeV}, \quad M_{Z^{\prime}}=2.3 \mathrm{TeV}$ for the LHC. Dash-dotted line corresponds to the SM, solid line corresponds to the sum $\mathrm{SM}+\mathrm{A}^{\prime}+\mathrm{Z}^{\prime}$, dashed line corresponds to the sum $\mathrm{SM}+\mathrm{A}^{\prime}+\mathrm{Z}^{\prime}+\mathrm{KK}$.

The cross-sections of these processes can be obtained in the same way, as those with the excitations of the $W$ boson. The distributions in the invariant mass of the $t \bar{t}$ pair and in the transverse momentum of the top quark are shown in figures 9 and 10 for $M_{\gamma}^{\prime}=2 \mathrm{TeV}, \quad M_{Z}^{\prime}=2.3 \mathrm{TeV}$ and in figures 11 and 12 for $M_{\gamma}^{\prime}=3 \mathrm{TeV}, \quad M_{Z}^{\prime}=3.4 \mathrm{TeV}$. 


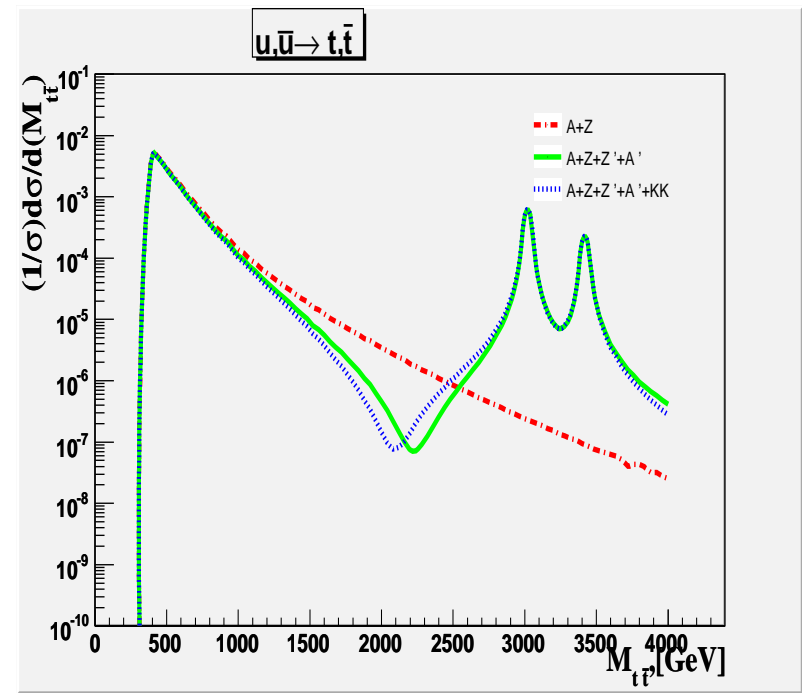

Figure 11: Distribution in the invariant mass of $t \bar{t}$ pair for $M_{\gamma^{\prime}}=3 \mathrm{TeV}, \quad M_{Z^{\prime}}=3.4 \mathrm{TeV}$ for the LHC. Dash-dotted line corresponds to the SM, solid line corresponds to the sum $\mathrm{SM}+\mathrm{A}^{\prime}+\mathrm{Z}^{\prime}$, dashed line corresponds to the sum $\mathrm{SM}+\mathrm{A}^{\prime}+\mathrm{Z}^{\prime}+\mathrm{KK}$.

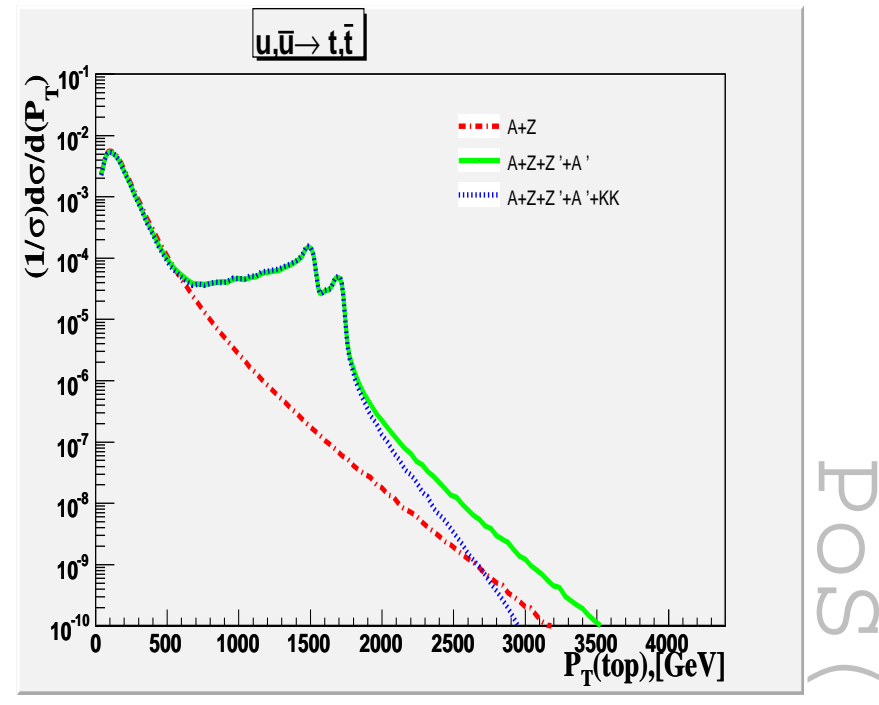

Figure 12: Top quark $p_{t}$ distribution for $M_{\gamma^{\prime}}=$ $3 \mathrm{TeV}, \quad M_{Z^{\prime}}=3.4 \mathrm{TeV}$ for the LHC. Dash-dotted line corresponds to the SM, solid line corresponds to the sum $\mathrm{SM}+\mathrm{A}^{\prime}+\mathrm{Z}^{\prime}$, dashed line corresponds to the sum $\mathrm{SM}+\mathrm{A}^{\prime}+\mathrm{Z}^{\prime}+\mathrm{KK}$.

The distributions in the invariant mass of the $\mu^{+} \mu^{-}$pair and in the transverse momentum of the muon are shown in figures 13 and 14 for $M_{\gamma}^{\prime}=2 \mathrm{TeV}, \quad M_{Z}^{\prime}=2.3 \mathrm{TeV}$.

\section{$\mathrm{p} p \rightarrow \mu^{+} \mu^{+}+\mathrm{X}$}

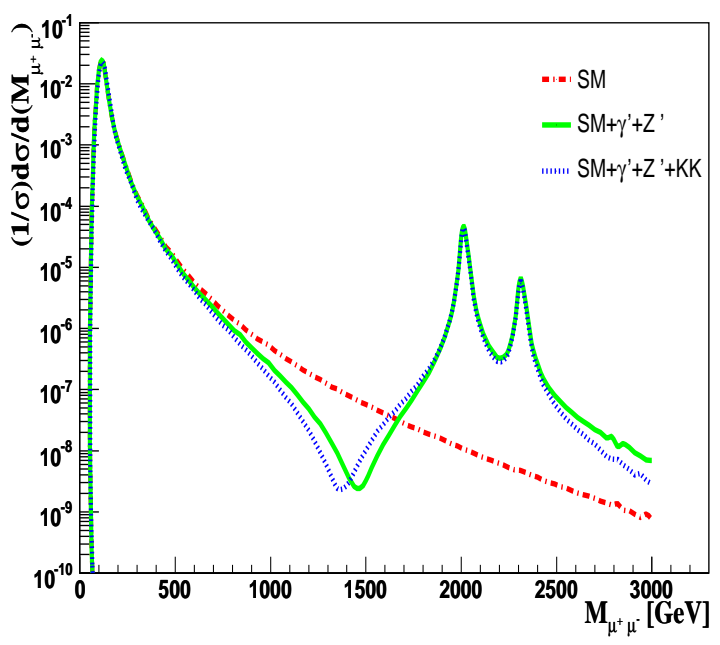

Figure 13: Dilepton invariant mass distribution for $M_{\gamma^{\prime}}=2 \mathrm{TeV}, \quad M_{Z^{\prime}}=2.3 \mathrm{TeV}$ for the LHC. Dashdotted line corresponds to the SM, solid line corresponds to the sum $\mathrm{SM}+\mathrm{A}^{\prime}+\mathrm{Z}^{\prime}$, dashed line corresponds to the sum $\mathrm{SM}+\mathrm{A}^{\prime}+\mathrm{Z}^{\prime}+\mathrm{KK}$.

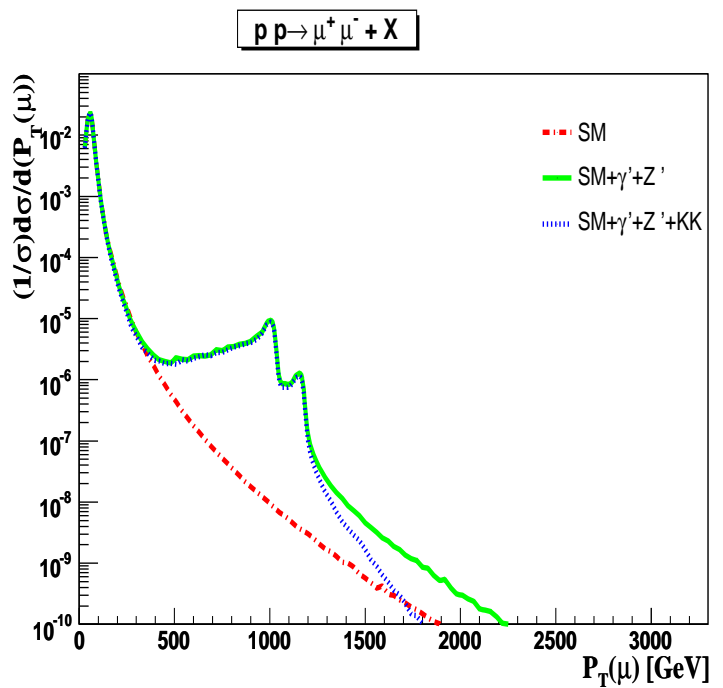

Figure 14: Dilepton $p_{t}$ distribution for $M_{\gamma^{\prime}}=$ $2 \mathrm{TeV}, \quad M_{Z^{\prime}}=2.3 \mathrm{TeV}$ for the LHC. Dash-dotted line corresponds to the SM, solid line corresponds to the sum $\mathrm{SM}+\mathrm{A}^{\prime}+\mathrm{Z}^{\prime}$, dashed line corresponds to the sum $\mathrm{SM}+\mathrm{A}^{\prime}+\mathrm{Z}^{\prime}+\mathrm{KK}$. 
We have to note here, that the case of the $Z^{\prime}$ boson and $\gamma^{\prime}$ turns out to be more complicated, because in theories with extra dimensions the KK graviton $g r^{\prime}$ and its tower also contribute to all the processes mediated by the neutral vector boson. (To be precise, there is one more tower that contributes to all these processes, namely that of the scalar radion. But the contribution of the scalar modes is suppressed by the factor $\left(m_{q} / M\right)^{2}, m_{q}$ being the mass of a first generation quark and $M$ being the fundamental energy scale of the order of several $\mathrm{TeV}$ [19]. For this reason the contribution of the scalar modes is negligible and we discard it completely.) The contribution of the processes with the first graviton excitation and its KK tower to $\mu^{+} \mu^{-}$pair production is described by the following diagrams, which are not summed coherently:
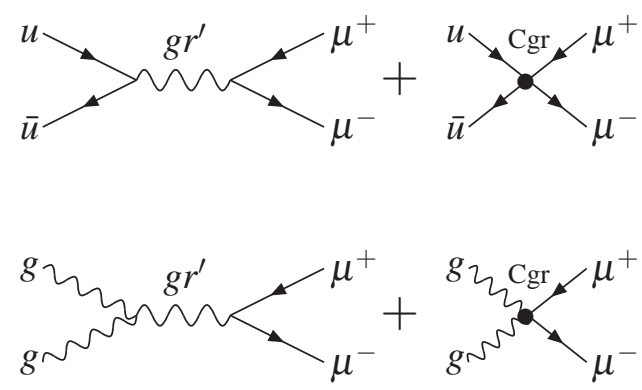

The results of the calculations with the inclusion of these diagrams of the distributions in the invariant mass of the $\mu^{+} \mu^{-}$pair and in the transverse momentum of the muon are shown in figures 15 and 16 for $M_{\gamma}^{\prime}=2 \mathrm{TeV}, \quad M_{Z}^{\prime}=2.3 \mathrm{TeV}$ and $M_{g r^{\prime}}=3.83 \mathrm{TeV}$.

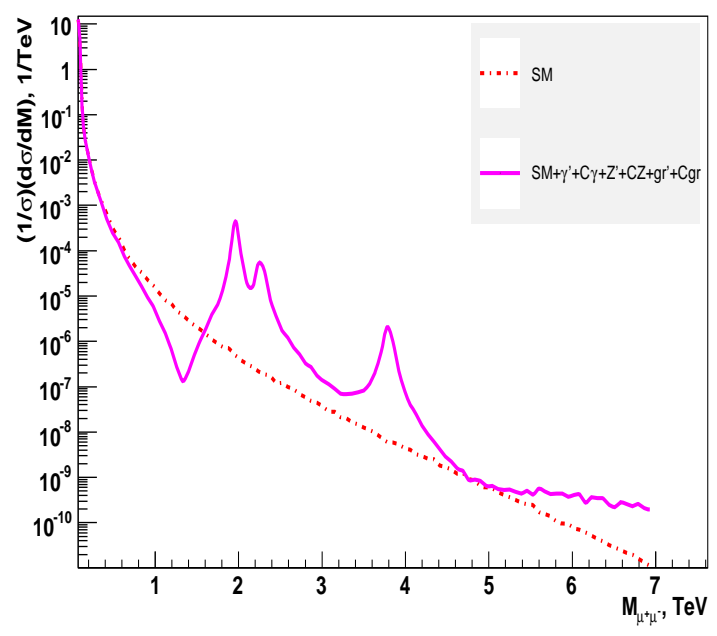

Figure 15: Dilepton invariant mass distribution for $M_{\gamma^{\prime}}=2 \mathrm{TeV}, \quad M_{Z^{\prime}}=2.3 \mathrm{TeV}$ and $M_{g r^{\prime}}=3.83 \mathrm{TeV}$ for the LHC. Dash-dotted line corresponds to the SM, solid line corresponds to the sum SM+ A'+ Z' + gr' + KK.

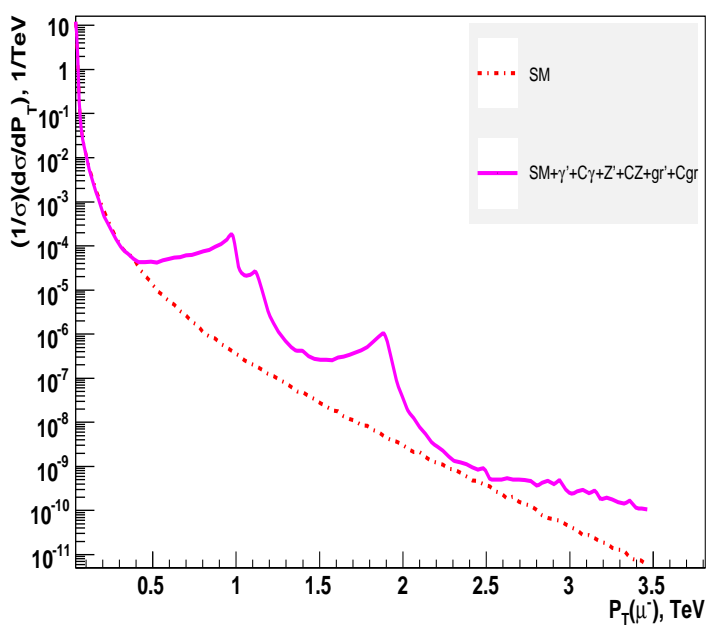

Figure 16: Dilepton $p_{t}$ distribution for $M_{\gamma^{\prime}}=$ $2 \mathrm{TeV}, \quad M_{Z^{\prime}}=2.3 \mathrm{TeV}$ and $M_{g r^{\prime}}=3.83 \mathrm{TeV}$ for the LHC. Dash-dotted line corresponds to the SM, solid line corresponds to the sum $\mathrm{SM}+\mathrm{A}^{\prime}+\mathrm{Z}^{\prime}+\mathrm{gr}{ }^{\prime}+\mathrm{KK}$. 


\section{Conclusion}

Theories with extra dimensions have a long history marked by alternating periods of oblivion and keen interest. The latest developments in this field abolished the old restrictions on the size of the extra dimensions, and now their predictions can be confronted with the experiment.

Since in higher-dimensional Einstein gravity it is impossible to find braneworld solutions with more that two extra dimensions, we believe that nowadays the most consistent model with extra dimensions is the stabilized Randall-Sundrum model. If its fundamental parameters lie in the $\mathrm{TeV}$ energy range for the observer on the negative tension brane, the effects due to the massive modes can be observed in collider experiments. If the masses of the KK gravitons and of the excitations of the SM fields are beyond the energy range of direct observation, the interactions due to KK towers can be well approximated by contact interactions of the type of Lagrangian (5.2) or of Fermi's four-fermion Lagrangian, the Tevatron data demanding the coupling constant $\Lambda_{\pi}$ to be larger than $610 \mathrm{GeV}$. If these masses are in the energy range of direct observation, the effective contact interaction induced by the infinite towers of the massive gravitons or of the KK excitations of the SM particles should also be taken into account to correctly look for KK resonances and to model the distribution tails. An observation of the interference between the first KK graviton or SM particle resonance and the rest of its KK tower should be considered as a strong argument in favor of the extra dimensions hypothesis.

\section{References}

[1] Bernhard Riemann, On the Hypotheses which lie at the Bases of Geometry (translated by William Kingdon Clifford), Nature 8 14-17, 36, 37 (1873)

[2] G. Nordström, Über die Möglichkeit, das elektromagnetische Feld und das Gravitationsfeld zu vereinigen, Physikalische Zeitschrift 15 504-506 ( 1914) [physics / 0702221 ].

[3] T. Kaluza, Zum Unitätsproblem in der Physik, Sitzungsber. Preuss. Akad. Wiss, Berlin, Math. Phys. H1, 966-972 (1921).

[4] O. Klein, Quantentheorie und fünfdimensionale Relativitätstheorie, Z. Phys. 37, 895-906 (1926)

[5] R. Kerner, Generalization of the Kaluza-Klein theory for an arbitrary nonabelian gauge group. Annales Poincare Phys. Theor. 9, 143 (1968).

[6] Yu.A. Kubyshin, I.P. Volobuev, J. M. Mourao and G. Rudolph, Dimensional reduction of gauge theories, spontaneous compactification and model building Lecture Notes in Physics, 349 (1990)

[7] V.A. Rubakov, M.E. Shaposhnikov, Do We Live Inside A Domain Wall? Phys. Lett. B125 (1983) 136

[8] V.A. Rubakov, M.E. Shaposhnikov, Extra Space-Time Dimensions: Towards A Solution To The Cosmological Constant Problem, Phys. Lett. B125 (1983) 139

[9] N. Arkani-Hamed, S. Dimopoulos, G. Dvali, The hierarchy problem and new dimensions at a millimeter, Phys. Lett. B429 (1998) 263

[10] L. Randall, R. Sundrum, A large mass hierarchy from a small extra dimension, Phys. Rev. Lett. 83 (1999) 3370

[11] M. N. Smolyakov, Submanifolds in space-time with unphysical extra dimensions, Class. Quantum Grav. 25 (2008) 238003 
[12] W. D. Goldberger, M. B. Wise. Modulus stabilization with bulk fields, Phys. Rev. Lett. 1999. V. 83. P. 4922.

[13] O. DeWolfe, D. Z. Freedman, S. S. Gubser, A. Karch. Modeling the fifth dimension with scalars and gravity, Phys. Rev. D. 2000. V. 62. P. 046008.

[14] E. E. Boos, Yu. S. Mikhailov, M. N. Smolyakov, I. P. Volobuev, Physical degrees of freedom in stabilized brane world models, Mod. Phys. Lett. A 21, 1431-1449 (2006).

[15] V. M. Abazov et al. [The D0 Collaboration], Search for Randall-Sundrum gravitons in the dielectron and diphoton final states with $5.4 \mathrm{fb}-1$ of data from ppbar collisions at $\operatorname{sqt}(\mathrm{s})=1.96 \mathrm{TeV}$, Phys. Rev. Lett. 104, 241802 (2010) [hep-ex/1004.1826].

[16] Sean M. Carroll, Monica M. Guica, Sidestepping the cosmological constant with football shaped extra dimensions, hep-th/0302067

[17] D. Lovelock, J. Math. Phys. 12 (1971) 498.

[18] C. Charmousis and R. Zegers, JHEP 0508 (2005) 075 [hep-th/0502170].

[19] E.E. Boos, V.E. Bunichev, M.N. Smolyakov and I.P. Volobuev, Testing extra dimensions below the production threshold of Kaluza-Klein excitations, Phys.Rev. D79 104013 (2009) [hep-ph/0710.3100v4]

[20] E. Boos et al. [CompHEP Collaboration], CompHEP 4.4: Automatic computations from Lagrangians to events, Nucl. Instrum. Meth. A 534250 (2004) [hep-ph/0403113].

[21] E. E. Boos, M. A. Perfilov, M. N. Smolyakov and I. P. Volobuev, Theor. Math. Phys. 170 (2012) 90.

[22] J. A. M. Vermaseren, New features of FORM, math-ph/0010025;

J. A. M. Vermaseren, Prospects of FORM, Nucl. Instrum. Meth. A 559 1-5 (2006) . 